\title{
8
}
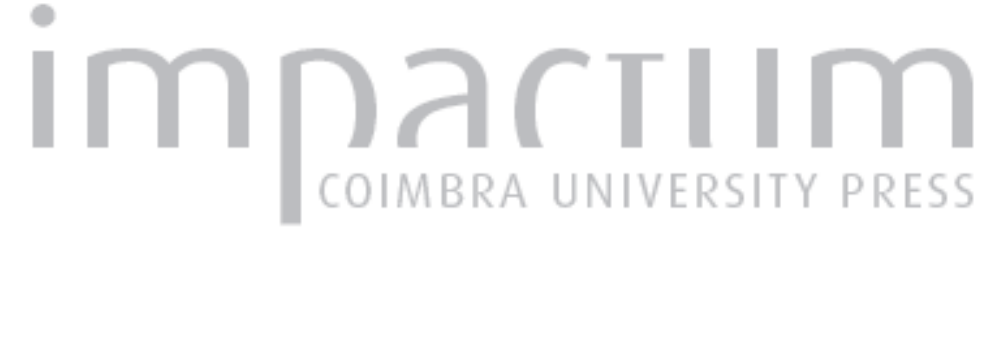

\section{Gestos, palabras y actitudes en el De facie in orbe Lunae de Plutarco}

Autor(es): Pérez Jiménez, Aurelio

Publicado por: International Plutarch Society

URL persistente:

URI:http://hdl.handle.net/10316.2/37575

DOI:

DOI:http://dx.doi.org/10.14195/0258-655X_1_5

Accessed : $\quad$ 26-Apr-2023 07:25:23

A navegação consulta e descarregamento dos títulos inseridos nas Bibliotecas Digitais UC Digitalis, UC Pombalina e UC Impactum, pressupõem a aceitação plena e sem reservas dos Termos e Condições de Uso destas Bibliotecas Digitais, disponíveis em https://digitalis.uc.pt/pt-pt/termos.

Conforme exposto nos referidos Termos e Condições de Uso, o descarregamento de títulos de acesso restrito requer uma licença válida de autorização devendo o utilizador aceder ao(s) documento(s) a partir de um endereço de IP da instituição detentora da supramencionada licença.

Ao utilizador é apenas permitido o descarregamento para uso pessoal, pelo que o emprego do(s) título(s) descarregado(s) para outro fim, designadamente comercial, carece de autorização do respetivo autor ou editor da obra.

Na medida em que todas as obras da UC Digitalis se encontram protegidas pelo Código do Direito de Autor e Direitos Conexos e demais legislação aplicável, toda a cópia, parcial ou total, deste documento, nos casos em que é legalmente admitida, deverá conter ou fazer-se acompanhar por este aviso.

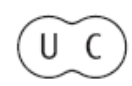




\title{
Gestos, PALABRas y ACTITUdeS en el De facie in orbe Lunae de Plutarco ${ }^{1}$ por \\ Aurelio Pérez Jiménez Universidad de Málaga
}

\begin{abstract}
In this article, we offer an analysis of some compositional features in the first part of Plutarch's dialog De facie in orbe Lunae. Firstly, we propose a stylistic use of the verbal forms $\epsilon \hat{\imath} \pi \nu \nu, \epsilon i \pi \epsilon / \epsilon \emptyset \eta(\nu)$ to distinguish the principal and the secondary characters, so as to point out the nuances of their interventions; secondly, we essay to elucidate the addressees of Lucius' and Lamprias' principal expositions; and, thirdly, we underline the importance of some dramatic elements in the structure of the diatribe. We can imagine the discussion in this dialogue as a lively debate, where not only the words, but also the speakers' attitudes and gestures play an important role. Through them, Plutarch lets us know -sometimes ironically- his philosophical sympathies and antipathies, giving us enough proofs of his ability to make a precise characterization of his literary personages.
\end{abstract}

\section{1}

El diálogo De facie in orbe Lunae de Plutarco tiene unas características que lo hacen especial dentro de la obra del Queronense. Lamprias, el hermano de Plutarco, cuenta a alguien una conversación cuyo objeto principal era conocer el mito de Sila, referente al papel cósmicometafísico de la Luna, como sede de los démones y lugar intermedio en el proceso de la generación y en el destino esca- tológico del hombre. Naturalmente la estrella invitada de este diálogo es Sila, aunque el relato viene precedido por la descripción, a cargo también de Lamprias, de los resultados de una disertación por un platonista sobre las explicaciones científicas y vulgares de las manchas que aparecen en la cara de la Luna. Este resumen dará pie a la intervención de los distintos personajes presentes en el diálogo y que son, además de Sila y de Lamprias, un pitagórico etrusco, Lucio, un gramático próximo a Lamprias y Lucio, Teón de Egipto, experto en cuestiones literarias, un peripatético, Aristóte-

Ofrecemos aquí una versión revisada y ampliada del trabajo presentado en el encuentro de Montpellier de la Red Europea de Plutarco en septiembre de 2000. Agradezco al Prof. Vicente Ramón Palerm la lectura previa del trabajo y sus sugerencias. El mismo forma parte del Proyecto BFF 2001-1893, financiado por la DGESIC. 
les, un estoico, Fárnaces, un geómetra entendido en óptica y próximo también a las posiciones del estoicismo, Apolónides el Táctico, y un matemático, Menelao, al que se le suponen conocimientos suficientes de astronomía.

Puesto que el personaje principal es, como decíamos, Sila, Plutarco estructura el diálogo tomando como referencia la razón de ser de su participación en el diálogo, que es contar el mito que oyó de un extranjero, servidor de Crono. Pues bien, de acuerdo con esto, la obra puede dividirse en tres partes, que suponen tres tipos diferentes de contenido: la primera (caps. $1-23=920 \mathrm{~B}-937 \mathrm{D})^{2}$ contiene la exposición, a cargo de Lamprias y de Lucio, de la diatriba en la que el ÉTaĩpos trató las doctrinas filosóficas y científicas sobre la naturaleza de la Luna, para explicar las manchas de su cara; la segunda (caps. $24-25=937 \mathrm{D}-940 \mathrm{~F})^{3}$ aporta nuevos datos a la diatriba en cuanto que aquí se ofrecen hipótesis (apoyadas en documentos literarios sobre todo y en la competencia en este terreno de Teón) relativas a la posibilidad de algún tipo de vida en la Luna, condición importante para la doctrina del mito; y la tercera (caps. $26-30=940 \mathrm{~F}-945 \mathrm{D}$ ) recoge el mito de Sila ${ }^{4}$.

Por supuesto el giro temático más violento del diálogo se produce con la decisión de pasar de la discusión científica, agotada ya en sus resultados racionales, a escuchar la historia mítica (y por tanto explicación religiosa) de Sila. Como sucede en otros diálogos parecidos de Plutarco, la conversación previa, con un narrador principal (Lamprias en este caso), pero salpicada de intervenciones pequeñas, preguntas y respuestas, de los otros participantes en el diálogo, con interrupciones más o menos bruscas, con ironías y desconfianzas que delatan las diferencias doctrinales de los interlocutores, pese a que su hilo conductor es el de las posibilidades analíticas de la razón (o precisamente por ello), tiene lugar paseando, una actitud adecuada para la poca concentración que exige su contenido. La conclusión de esta primera parte, que deja insatisfechos a parte de los interlocutores (Fárnaces y Apolónides) es la

2 Es una parte muy interesante para la historia de la astronomía lunar, cuyos problemas e interpretaciones pueden leerse en el comentario de H. GöRGEMANNS, 1972, espec. pp. 6278, así como en el magnífico análisis de L. TORRACA, 1992 y el de M. BALDASSARI, 1992.

Véase sobre la función literaria de esta parte, así como sobre los esfuerzos por identificar las manchas con relieves similares a los de la tierra, que fundamenten su habitabilidad, A. PÉREZ JimÉNEZ, 1991. Nuevas precisiones sobre el valor literario de esta parte en B. VAN MEIRVENNE, 2001.

4 Ésta, que queda al margen de nuestras consideraciones en la presente ocasión, ha sido la parte más atractiva para la investigación moderna, preocupada sobre todo por las cuestiones demonológicas y escatológicas del De facie. 
naturaleza térrea de la Luna ${ }^{5}$, con la que se dan por terminados los argumentos expuestos en la conferencia del ÉTaĩpos. Ahora ya Lamprias, satisfecha la curiosidad de Sila, propone oír el mito de éste, objeto principal de la reunión que motivó el diálogo. Para ello sugiere un cambio de actitud que es significativo:

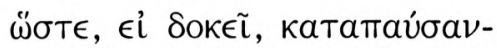

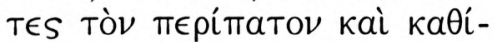

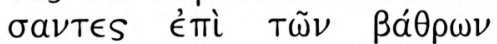

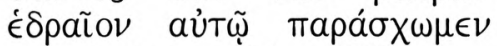

$$
\begin{aligned}
& \text { áкроатท́рเоv (24 = 937D). }
\end{aligned}
$$

Este cambio de actitud, que simboliza a un tiempo un mayor interés, curiosidad y concentración por parte de los oyentes respecto a lo que se va a contar, sirve a Plutarco, como en otras ocasiones ${ }^{6}$, para elevar la importancia de la hipótesis teleológica y de la explicación religiosa, escatológica, del tratado, que no es sino una exposición de parte de la demonología, tan importante para el Queronense.

En efecto, la disertación del éTaĩpos, resumida por Lamprias (y Lucio) a petición de Sila, como algo pertinente para su historia, se limitaba a discutir las doctrinas filosófico-científicas sobre la naturaleza de la Luna y a concluir que ésta era térrea. Falta en este resumen de Lamprias algo que parece fundamental para el mito, a saber, el sentido teleológico de aquella conclusión científica. La intervención de Teón cumple, pues, y así lo hemos hecho notar en otra parte $^{7}$, dos funciones:

1) Desde el punto de vista del tema, sirve como nexo necesario entre la parte científica (que la Luna es térrea) y el mito (que allí habitan los démones), puesto que establece la razón de ser de esa naturaleza térrea, a saber, su habita-

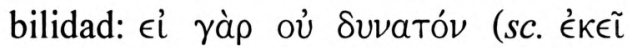

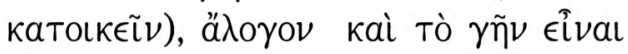
Tìv $\sigma \in \lambda \eta ́ v \eta \nu$ (937D,Teón) $\mathrm{y}$, en todo caso, sienta el papel intermedio de la Luna en el proceso de la generación y en la escatología (Lamprias).

2) $Y$ desde el punto de vista literario, la ausencia de dogmatismo a que da pie el tema (pura hipótesis), la gracia y el desenfado con que lo trata Teón y las referencias literarias y mitológicas que contiene, rebajan la tensión provocada por la discusión científico-filosófica y concentran la atención de todos los interlocutores en el relato de Sila. Así, al menos, parece deducirse de las palabras de Lamprias:

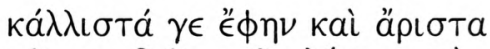

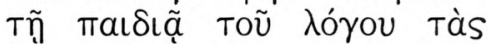

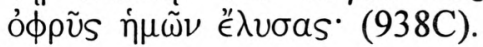

Doctrina de dudosa filiación platónica, aunque es defendida como tal por Lamprias. La naturaleza térrea de la luna cuenta con más apoyo en la tradición pitagórica, por lo que parece presumible que pertenezca a la tesis de Lucio ( $c f$. sobre el tema H. GöRGEMANNS, 1972, pp. 34-39).

Def. orac. 412D, Sept. sap. conv. 149F, E ap. Delph. 385A-B y Non poss. suav. viv. 1100E.

7 1991, pp. 310-311. 
Es, por tanto, el diálogo, entre dos personajes que sintonizan perfectamente (y que representan ambos el pensamiento de Plutarco), un paso intermedio para conseguir el silencio religioso (la $\in \dot{\phi} \phi \eta$ $\mu(a)$ que requiere el relato de Sila.

El giro de la conversación y la condición de los temas parece que no deja lugar a dudas respecto a esas tres partes propuestas para la estructura del diálogo. Pero, ya que el objeto de nuestro trabajo en esta ocasión es más literario y formal que de contenidos, permítasenos una observación que, a nuestro juicio, apunta también en la misma dirección. Tal vez sea casual, pero en los numerosos cortes que tiene el relato de Lamprias, por intervención de Aristóteles, Lucio, Fárnaces, Apolónides y Teón, sobre todo -aunque éste, en algún momento, se dirige a cada uno de ellos por

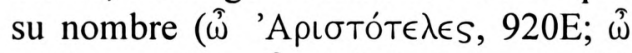

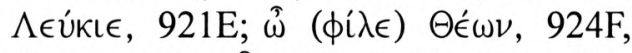

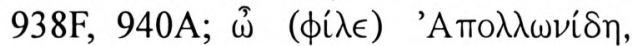

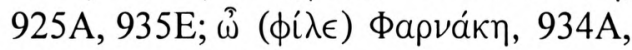
934C)-, aquéllos nunca pronuncian el nombre de Lamprias en sus respuestas o en sus preguntas. Sin embargo, y esto nos parece significativo, tanto la entrada de Teón, que inicia la segunda parte, como la interrupción brusca de Sila, con que comienza el relato del mito, y su alo- cución final, vienen precedidas por un $\hat{\omega}$ $\Lambda \alpha ́ \mu \pi \rho \iota a$ (937D y 940F, 945D respectivamente) que descubren la identidad del narrador del diálogo y dan un evidente protagonismo primero a Teón, proponente del nuevo tema (la teleología de la Luna) y luego a Sila, que recupera así de forma definitiva su papel en el diálogo.

Sentados estos principios, veamos ahora de qué modo la dinámica del diálogo, los gestos, las palabras y actitudes de los interlocutores se van ajustando a la personalidad de aquéllos y a la naturaleza e intensidad de las ideas y de las posiciones ideológicas que representan.

\section{2}

Puesto que el tratado que comentamos es un diálogo de estilo platónico ${ }^{8}$ de segundo nivel, me parece pertinente decir algo, en primer lugar, sobre los verbos con que el narrador, Lamprias, introduce a sus personajes y que son, como señala el profesor Ureña Bracero para otros diálogos ${ }^{9}$, las formas habitua-

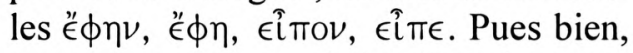
en nuestro diálogo, dado el relativamente alto número de interlocutores (siete en el uso de la palabra), el gran número de interrupciones (contamos salvo error $u$ omisión 34 intervenciones directas,

8 Similar al Banquete o al Fedón (cf. D. DEL CORNO, 1991, pp. 27-28 y V. RAMÓn PALERM, 2002, p. 122).

9 Cf. J. UREÑa BraCERO, 1996, pp. 32 ss. Jesús Ureña observa cómo en los diálogos plutarqueos se establece una clara tendencia al uso de $\epsilon^{\prime \prime} \phi \eta \nu, ~ " ̈ \phi \eta$ por los personajes principales frente a $\epsilon i \pi \epsilon$ para referirse a los personajes secundarios, lo que coincide en gran medida, como veremos, con nuestros datos. 
algunas de ellas con hasta 3 cambios de interlocutor) y la amplitud de algunos discursos, cuya monotonía se rompe a veces con estas fórmulas verbales, hay un total de veintitrés usos de $\epsilon$ îtTov (sólo en siete ocasiones el narrador principal recurre a esta forma), $\epsilon i \pi \epsilon$ y diecinueve de $\epsilon € \phi \eta \nu$ (en diez ocasiones utilizado por Lamprias), $\dddot{\epsilon} \phi \eta$. Sin embargo, la distribución de ambas formas no parece fortuita ni carente de interés, por su pertinencia respecto a la importancia de los personajes introducidos con uno $u$ otro verbo. Analicemos, aunque sea sucintamente, los datos:

El narrador, Lamprias, utiliza $€ \notin \phi \nu$, $\epsilon \emptyset \eta$ para introducir a los siguientes personajes: Él mismo (diez veces), Apolónides (dos veces) y Lucio (siete veces).

En cambio, utiliza $\epsilon \hat{\imath} \pi 0 \nu, \epsilon \hat{\imath} \pi \epsilon$, con referencia a sí mismo, siete veces, a $\mathrm{Si}$ la, cinco, a Apolónides, una, a Lucio, cuatro, a Fárnaces, tres, a Aristóteles, una y a Teón, dos.

De estos datos deducimos que Plutarco reserva el imperfecto de $\phi \eta \mu i$ para Lamprias y Lucio, los personajes sobre cuyos hombros recae todo el peso de la discusión científica y que son, en general, portavoces de la opinión del autor. De hecho, aunque la tarea de contar a los presentes la conferencia del étaĩoos es asumida casi en su totalidad por Lamprias, en alguna ocasión no duda (cuando se trata de la crítica a los estoicos) en pasarle la palabra a Lucio. En cambio, los demás personajes son introducidos por el verbo $\epsilon \hat{\imath} \pi \epsilon$ que, de alguna manera, marca la brevedad o la naturaleza de sus interrupciones, tomas de posición o res- puestas provocadas por el discurso de alguno de los dos personajes principales y que denota su papel secundario en la discusión científica. No obstante, hay excepciones a esta regla que, a mi juicio, son a menudo susceptibles de una explicación estilística: $\epsilon \hat{\imath} \pi 0 \nu$, en 920B, es una réplica muy ligada a la propuesta de Sila (que dice que le gustaría conocer antes de contar su relato las opiniones usuales sobre la cara de la luna) y, por tanto, con una fuerte carga impresiva, que justifica el aoristo. Lo mismo es válido para la respuesta inmediata de Lamprias a la pregunta de Apolónides (921B) sobre la refutación de Clearco. También está cargada de afectividad la frase (introducida de igual modo con el aoristo) con la que Lamprias en cierto modo recrimina a Lucio su diplomático uso de los términos al referirse a los estoicos; de hecho su frase es una interrupción del discurso de aquél: $\chi \rho \eta \sigma \tau \tilde{\omega} s \gamma^{\prime}$

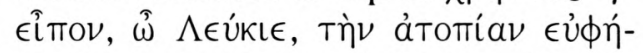

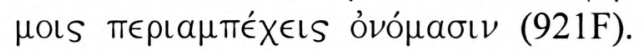
Por último, los otros 4 casos $(923 \mathrm{~F}$, 925A, 926C y 937C) pertenecen igualmente más al lenguaje impresivo que al discursivo, por cuanto en el primero -en un contexto en el que Lamprias quiere dar tiempo a Lucio para que piense su respuesta a los argumentos de Fárnacesla pregunta a Teón sobre quién es el au-

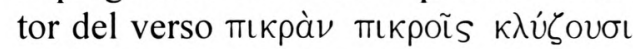
фарна́когs ходńv es una maniobra de distracción que sirve además, a Lamprias, para cargar sus baterías contra los estoicos; pues bien, la respuesta de Teón

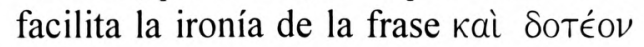

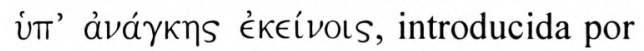


$\epsilon \hat{\imath} \pi \mathrm{v}$, con que Lamprias descarga doblemente esas baterías. En el segundo, la ironía que justifica el uso del aoristo (en un contexto de discusión de la doctrina estoica, aunque se dirija a Apolónides) viene marcada (como veremos después) por el uso del adjetivo $\phi i ́ \lambda \epsilon$ en la fórmu-

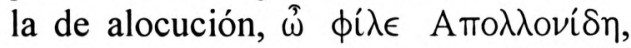
empleada. En el tercero, la frase introducida, en la que figura la exclamación mpòs $\Delta$ tós, posiciona definitivamente a Lamprias en la crítica sarcástica a Fárnaces. Y en el cuarto (último argumento para rebatir la opinión de los estoicos) el aoristo (no era necesario ni el aoristo ni el imperfecto) reafirma la posición de Lamprias de la que quiere hacer partíci-

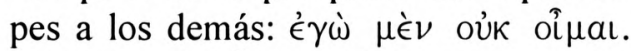

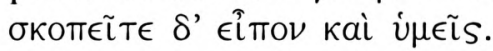

La misma interpretación cabe dar al uso de $\epsilon \hat{\imath} \pi \epsilon$ en las cuatro ocasiones en que aparece referido a Lucio: $922 \mathrm{~F}$ es clarísimo: Lucio no puede soportar la interrupción de Fárnaces e interviene súbitamente $(\epsilon \hat{\imath} \pi \epsilon \nu)$ con burla y sarcasmo. En 927E, con el aoristo, se marca la interrupción a Aristóteles, que, con su entrada, ha retrasado la intervención de Lucio solicitada por Lamprias, así como una cierta dosis de afectividad con que se quiere restar el disimulado enfado del peripatético. En 930A, el valor impresivo de las palabras con que Lucio corta a Sila, implícito en la construcción sintáctica (y marcado con el aoristo $\epsilon \hat{\imath} \pi \epsilon$ ) se refuerza con la interjección: $\dot{\alpha} \lambda \lambda \grave{\alpha} \nu \grave{\eta}$

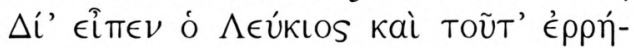
$\theta \eta$. Y por último, en $932 \mathrm{D}$, el uso del aoristo se justifica porque, con las palabras introducidas por él, Lucio aprueba complacido la respuesta de Lamprias: ...ỏo$\theta \tilde{\omega} s$ ' $\epsilon i \pi t \epsilon \nu$ 'iтté $\mu \nu \eta \sigma a s$.

En cuanto a los otros personajes, resul-

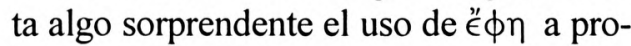
pósito de Apolónides - uno de los caracteres secundarios- en 935D y 936D.

El primer pasaje es especialmente significativo, ya que contraviene todo cuanto hemos dicho arriba. Se aplica a un personaje secundario y, al menos aparentemente, hay cierta carga impresiva, evidente en el empleo de la fórmula $\bar{\omega}$ mpòs aủTñs Tñs $\Sigma \in \lambda \eta \dot{\nu \eta \eta s . ~ A d e m a ́ s, ~ e l ~ v e r b o ~}$ viene acompañado de un participio, útro$\lambda \alpha \beta \omega v$, utilizado en otros pasajes para indicar las interrupciones. Esperaríamos, pues, más el aoristo que el imperfecto. No obstante, cabría hacer las siguientes precisiones: Su intervención no interrumpe realmente el discurso de Lamprias, sino que, más bien, es una toma de la palabra aprovechando un silencio del anterior. En su intervención -con la que él pretende, como técnico en astronomía, precisar algunos aspectos de la astronomía lunar- observamos cierta cautela, lo que puede implicar una merma de afectividad, acorde con su temperamento como científico. Eso explicaría una voluntaria elección por Plutarco en el uso del imperfecto en lugar del aoristo.

En el otro caso, 936D, Plutarco, con el uso igualmente del imperfecto, tal vez quiere decirnos que Apolónides está en calma respecto a Lamprias y que su pregunta no contiene segundas intenciones ni matices añadidos. Éstos sí que aparecen, en cambio, en 921B, donde la interven- 
ción del matemático deja ver en las palabras, y seguramente también en los gestos, su complacencia por la explicación de

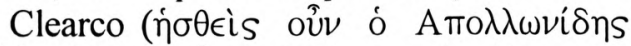

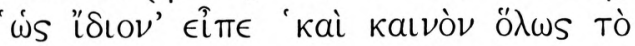

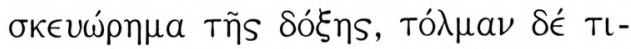

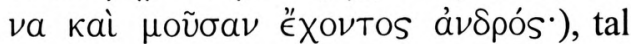
como la expone Lamprias en 920F-921A.

En definitiva, creemos que la especialización que, con carácter general, se observa en el diálogo (y en otros distintos) del imperfecto, reservado para el narrador o referido a los personajes que llevan la parte principal de la discusión con exposiciones más largas $\mathrm{y}$, por tanto, en las que domina lo discursivo sobre lo impresivo, viene justificada por la lógica calma que impone la ilación de los argumentos racionales en dichas intervenciones. Mientras que el uso del aoristo en estos casos y en las pequeñas entradas de los demás interlocutores -interrumpiendo, protestando, ironizando, o manifestando su complacencia u otros afectos-, denota la plasmación en el tono de voz, tal vez, de los estados de ánimo que acompañan las palabras.

El uso de estos verbos es, lógicamente, un procedimiento directo para generar cierta escenografía de la conversación. De hecho, lo hemos apuntado, con ellos no sólo se introducen las intervenciones de los personajes, sino que, en cierto modo, se cualifica la naturaleza de esa intervención, más pausada $(\varkappa € \eta[\nu])$ o más intensa ( $\epsilon \hat{\imath} \pi 0 \nu, \epsilon \hat{\imath} \pi \epsilon)$. Pero hay, a lo largo de las exposiciones, sobre todo de las más largas de Lamprias y Lucio, pronombres y paréntesis que nos guían e informan sobre la posición de los actores del diálogo. Por estos procedimientos hemos podido aislar, como dijimos, unas treinta y cuatro intervenciones diferentes, todas, salvo una (de Teón), introducidas en estilo directo mediante los verbos "ै $\phi \eta(\nu)$ y $\epsilon \hat{\imath} \pi \epsilon, \epsilon \hat{\imath} \pi 0 \nu$. Y, dentro de algunas de ellas, encontramos cambios de interlocutor, aunque no haya obligadamente respuesta. La situación respecto a estos cambios está menos clara y creo que requiere que nos detengamos en los pasajes en cuestión.

De acuerdo con estos indicadores, podemos recomponer la estructura del diálogo en la forma siguiente:

1) Discusión de la doctrina de Clearco sobre el reflejo del gran mar en la luna (920F-921E): El comienzo no plantea dudas: Lamprias se dirige a Aristóteles porque Clearco es un peripatético y expone sucintamente, a instancias de Apolónides (interesado como científico) la tesis óptica de Clearco. Apolónides interviene luego para mostrar su admiración por la originalidad de la doctrina y quiere saber cómo el étaĩ ${ }^{10}{ }^{10}$ rebatía

10 Cherniss (seguido en su traducción por V. RAMÓn PALERM, 2002, p. 132) acepta la con-

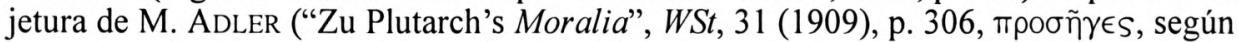
la cual Apolónides atribuiría la refutación de Clearco al propio Lamprias (ả $\lambda \lambda \grave{a} \pi \tilde{n}$ Tòv

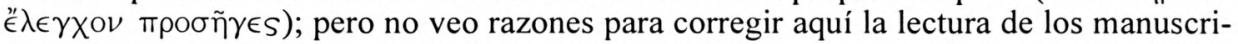
tos (véanse las objeciones de H. GörgemanNs, 1970, p. 42, n. 6 y de L. LeHNUS, 1991, p. 122, a la conjetura de Adler). 
sus argumentos. Entonces tiene lugar la respuesta de Lamprias y la dificultad de establecer los destinatarios. La primera

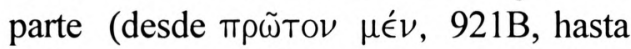
Emíkoupos, 921E) se dirige exclusivamente a Apolónides, como respuesta a su pregunta; abundan en el texto las referencias matemáticas (geometría y astronomía) y Lamprias manifiesta con cierto respeto su temor a tratar sobre la magnitud de la tierra en presencia del geómetra. El sin-

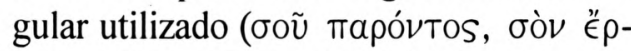
$\gamma \mathrm{ov}$ ) y estas referencias, además de la alusión a Hiparco, no deja lugar a dudas: Lamprias se dirige sólo a Apolónides. Cambia la situación, sin embargo, en la segunda parte (desde oủk $\dot{\epsilon} \theta \in \lambda \eta \dot{\sigma} \sigma \in \mathrm{l}$, 921E hasta $\dot{\alpha} \lambda \lambda^{\prime} . . ., 921 \mathrm{~F}$ ); el plural

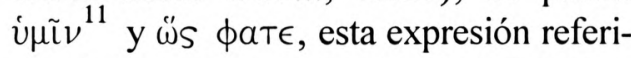
da a la doctrina peripatética de la luna como astro etéreo y con luz propia, inducen a creer que Lamprias se dirige ahora a Aristóteles o a éste.y a Apolónides (como observa Cherniss $^{12}$ ), cuyo posicionamiento con la tesis de Clearco puede venir implicado por el placer y admiración que despertaba en él su doctrina.

2.- Exposición por Lamprias de las críticas a la doctrina estoica sobre la Luna como un fuego latente $(921 \mathrm{~F}$ 922F): En esta intervención, dirigida a Lucio, hay que distinguir dos destinata- rios: el de la primera parte es Lucio (desde $\chi \rho \eta \sigma \tau \tilde{\omega} S, 921 \mathrm{~F}$, ¿hasta є̇ vov, 922D?). Que así es, queda claro no

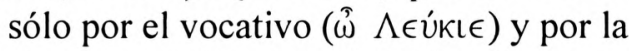

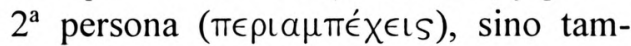

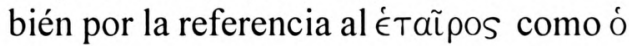
ÉTaĩpos $\dot{\eta} \mu \tilde{\omega} \nu$ y a los estoicos en tercera

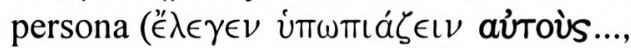
oíov oũTol....Toloũol, probablemente se-

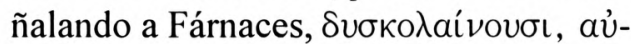

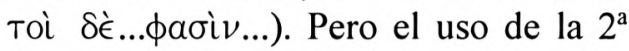
persona del plural ( ra referirse a los partidarios de la doctrina estoica, demuestra un cambio de destinatario a partir de cierto momento, que no está marcado en el texto. Pienso que ese giro de Lamprias hacia Fárnaces se

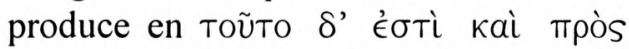

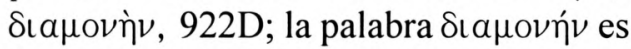
la clave, a mi juicio, para postular ese cambio de dirección. En efecto, en $922 \mathrm{~A}$, cuando se menciona la doctrina

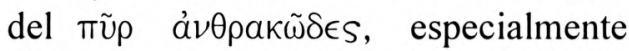
ligada a Fárnaces en nuestro diálogo, Lamprias le dice a Lucio que oủk ÉX $\in \mathrm{L}$

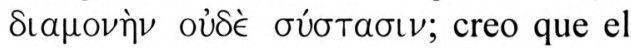
uso de la misma palabra denota la decisión de Lamprias de rebatir ahora directamente a los interesados su tesis.

3.- Irrupción de Fárnaces indignado: $\mathrm{Su}$ intervención $(922 \mathrm{~F})$ tiene también dos destinatarios distintos: La primera

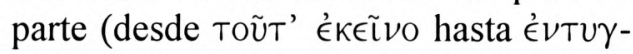

11 No vemos necesidad de corregir en $\eta \mu \tau \tilde{\nu}$, como hace CHERNISS, siguiendo a XYLANDER, KEPLER y WYTTENBACH (corrección aceptada en sus traducciones por L. LEHNUS, p. 53 y V. RAmÓN PAlerm, p. 134). El pronombre sería más un dativo de interés ("defender para vuestra escuela") que un comitativo ("defender con nosotros").

12

H. CHERNISS, 1951, pp. 137-138. 
$\chi a ́ v \omega \sigma \iota v)$ no tiene un interlocutor concreto. Es una explosión de ira, dirigida en todo caso al conjunto de los presentes, contra la Academia, cuyos representantes son aludidos en $3^{\mathrm{a}}$ persona: $\dot{\epsilon} \nu$

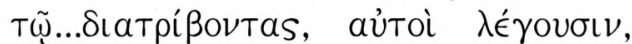

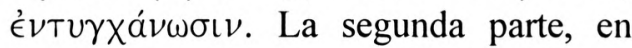
cambio, tiene como destinatarios concretos a Lamprias y a Lucio: '́é $\delta^{\prime}$ oûv

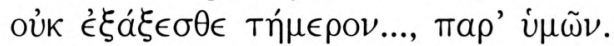

4.- Crítica de la doctrina estoica por parte de Lucio (922F-923E): Observamos en esta intervención al menos tres cambios de destinatario. La primera parte puede ser desde el comienzo ( $\mu$ óvov..., $\hat{\omega} T a ́ v, \ldots, 922 \mathrm{~F})$, hasta el final de la cita del Prometeo ( $\in$ úá $\gamma \kappa a \lambda o \nu, 923 \mathrm{C}$ ). Aquí el destinatario es Fárnaces, como demuestran los apelativos ( $\hat{\omega}$ Táv, $\hat{\omega}$ $\beta \epsilon ́ \lambda T L \sigma T \epsilon)$ y las segundas personas

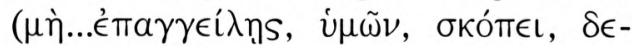

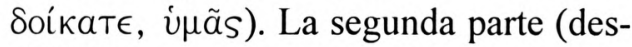

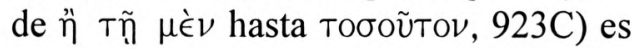
un inciso que Lucio dirige irónicamente hacia los demás compañeros, refiriéndo-

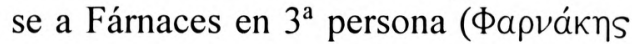
aủTòs... É $\sigma \tau \iota \nu$, oíkTí $\rho \in l)$. En la tercera

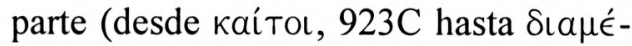
$\nu \in t \nu, 923 \mathrm{E})$ se recupera la crítica directa a Fárnaces con la exposición de las razones por las que no se cae la luna y por las que no puede ser fuego; la $2^{\mathrm{a}}$ persona vuelve a estar presente al final de la

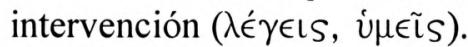

5.- Argumentos de Lamprias contra la tesis estoica (923F-927C): Es la intervención más larga de todo el diálogo y la que plantea mayores problemas respecto a la identificación de sus destinatarios directos. Comienza con una pregunta a Teón sobre el autor de un verso, y la respuesta de éste presentada en estilo indirecto. A partir de aquí la cuestión se complica. La

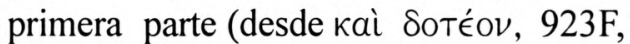

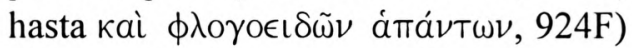
tiene como destinatario o bien al propio Teón o, lo que parece más probable, ya que Teón es sólo un mero pretexto para distraer la atención centrada en Lucio, a todos los presentes. Aquí los estoicos son aludidos en $3^{\mathrm{a}}$ persona $(\tilde{\sigma} \sigma \pi \epsilon \rho$

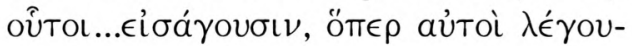

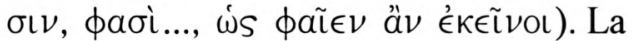
segunda parte (desde 'A $A \lambda$ ' hasta $\delta ı n \rho \tilde{\sigma} \sigma \theta a l, 926 \mathrm{~B})$ se dirige expresamente a Apolónides en virtud de su contenido sobre todo matemático y astronómico. La interpelación, iniciada con $\bar{\omega} \phi i ́ \lambda \epsilon$ 'A $A \pi \partial \lambda \omega \nu i \delta \delta$, se mantiene con las referencias a la $2^{\mathrm{a}}$ persona

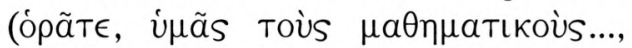

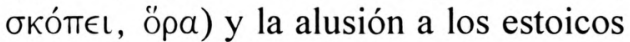

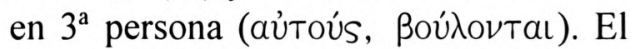
comienzo de la tercera parte es más discutible, pero creo que hay razones para

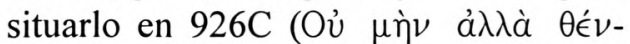
$T \in S$ ); el final podemos establecerlo en

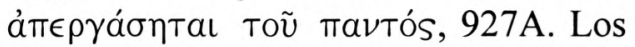
comentaristas suelen mantener como destinatario de esta parte (y de toda la exposición a partir de 925A) a Apolónides; pero esto nos parece a nosotros poco probable. Apolónides, igual que compartía opiniones con los peripatéticos, sin serlo, también coincide en ciertos momentos de la discusión con Fárnaces, pero no es en modo alguno un 
estoico. Pues bien, todo el contenido de esta parte está formado por principios estoicos que Lamprias discute o utiliza irónicamente y que atribuye a su interlocutor mediante el uso de la $2^{\mathrm{a}}$ persona

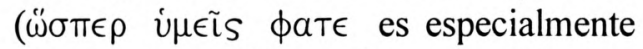

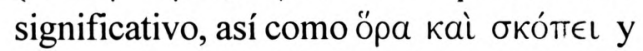
lo que sigue). Además, la frase $\mu \grave{\eta} \tau \rho \alpha$ -

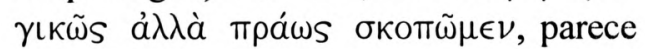
referirse irónicamente más a Fárnaces, cuya actitud airada se ridiculiza permanentemente en el diálogo, que a Apolónides. En el resto de la intervención, aunque siguen presentes los principios del estoicismo, es necesario suponer otro cambio de destinatario, ya que, al final (928C), se alude a los estoicos en $3^{\mathrm{a}}$ persona $(\epsilon \xi \xi \hat{\omega} \nu$

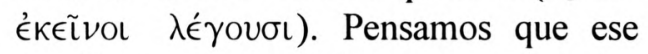
cambio se produce al comienzo del cap. 13 (927A) y que el destinatario vuelve a ser Apolónides, por la referencia a la táctica (927B). Que, dirigiéndose a Apolónides, utilice Lamprias los principios de los estoicos (como Zeus moınтทेs кaì тати́ $\rho$, y la relación entre macrocosmos y microcosmos) para demostrar la tesis de los académicos, no es sorprendente.

6.- Segunda intervención de Lucio (928F-929E): También en ésta debemos postular dos partes. La primera $(928 \mathrm{~F}-$ 929B) es la respuesta a Aristóteles explicando por qué la tesis académica ha centrado su debate en los estoicos sin hacer referencia a la doctrina aristotélica del movimiento circular y la composición divina de los astros. En esta parte el destinatario directo es Aristóteles, aludido por la $2^{\mathrm{a}}$ persona y el vocativo: $\dot{v} \mu \tilde{\imath} \nu, \hat{\omega}$

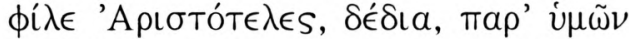

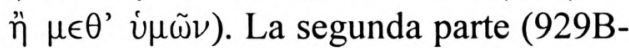
929E), en la que Lucio se propone exponer el resto de la diatriba, no tiene un destinatario concreto, sino el conjunto de los participantes en el diálogo.

7.- Tercera intervención de Lucio (930A-931C): Tras la pregunta de Sila, concerniente a si se trató la doctrina óptica sobre la Luna y tras su respuesta positiva, se dispone a explicarla. Las primeras palabras (930A) se dirigen al matemático Menelao, excusándose por adentrarse en cuestiones de óptica en su presencia

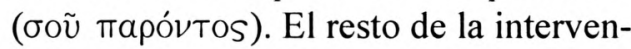
ción, cuya amplitud se corta con un $€$ $\phi \eta$ (930E), no tiene un destinatario concreto, sino el conjunto de los participantes

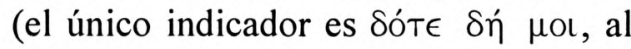
final de la exposición (931C) y el elogio de todos, al comienzo del cap. 19 (931D).

8.- Cuarta intervención de Lucio (931D-932C): La exposición, relativa a los eclipses, va dirigida también a todos los

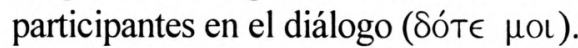

9.- Última intervención de Lucio (932E-933B): Demostración de la tesis académica teniendo en cuenta la sombra de los eclipses y crítica a los estoicos

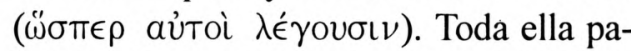
rece dirigida a Teón, a petición del cual se desarrolla. Dominan las segundas perso-

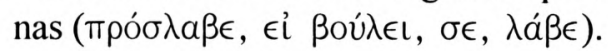

10.- Lamprias rebate la objeción de Apolónides relativa a la sombra (934AB) y sale al paso de la doctrina del fuego latente de Fárnaces (934B-934C): Aquí 
tenemos al menos tres partes, de las que la última no está bien marcada. La primera (934A) es la respuesta a Apolónides; pero nada indica que se dirija expresamente a Apolónides; puede hablar a todos los presentes. La segunda (934B-D) va dirigida a Fárnaces (mpòs

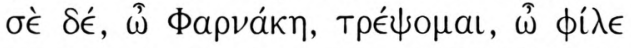

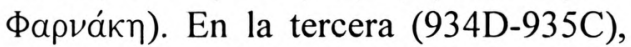
tal vez Lamprias, poco después de su última llamada a Fárnaces pasa a exponer su teoría del carácter térreo de la luna ante el conjunto de los presentes, anticipo de lo que será luego la tesis de Teón sobre la habitabilidad de la Luna.

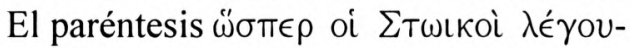
$\sigma \iota$ (935B) excluye que toda la argumentación se dirija a Fárnaces; y la referencia a quienes ven el color de la atmósfera durante los eclipses como de fuego latente, fundamento de la tesis de Fárnaces, nos inclina a pensar que a partir de aquí ya no está mirando Lamprias exclusivamente al estoico (934D: тобaútas

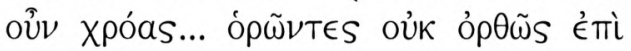

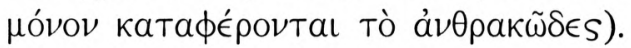

11.- La última intervención de Lamprias, en esta primera parte, va dirigida a

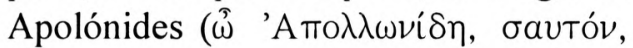
ol̈ $\in$,) que objeta la imposibilidad de que el tamaño relativo de las sombras haga posible interpretarlas como elementos del relieve lunar. Lamprias utiliza como argumento en contra de esta objeción las distorsiones ópticas producidas por la luz, lo que justifica que la intervención tenga como interlocutor directo a Apolónides, experto en esta ciencia. De hecho, en $936 \mathrm{C}$ vuelve a intervenir y Lamprias recupera la $2^{\mathrm{a}}$ persona para dirigirse a él: öpa; pero es posible que, enseguida, como en la respuesta a Fárnaces, pase a dirigir su tesis al conjunto de los presentes y no exclusivamente a Apolónides (tal vez con

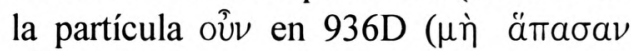

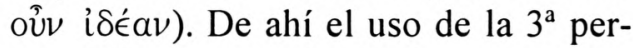
sona del plural para referirse a quienes utilizan la ciencia óptica en contra de

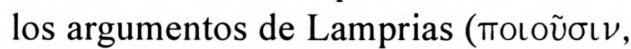

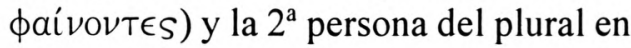
la parte última de su intervención, para referirse a sus oyentes (ópãte, 937B,

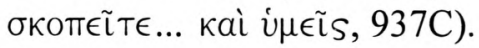

Pero no debemos olvidar otro elemento dramático que da vida a esas actitudes difícilmente captables muchas veces sólo por el contenido de las intervenciones. Me refiero a las invocaciones. Para empezar, y de acuerdo con lo que apuntábamos como posible intencionalidad al final del apartado anterior, las llamadas por nombre o por apelativos

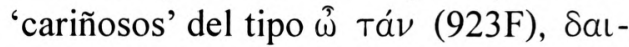

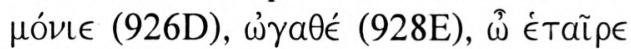
$(940 \mathrm{~A})^{12}$, se reservan exclusivamente a Lamprias y Lucio, que llevan todo el se dirigen ha sido subrayada para otros diálogos por J. UREÑa BRACERO, 1996, pp. 34-35. 
peso de la argumentación en el diálogo científico y, en los dos casos que dijimos, a Teón y a Sila, además de al extranjero en la última parte, que en dos ocasiones se dirige al cartaginés por su nombre: $\hat{\omega} \quad \Sigma u ́ \lambda \lambda \alpha$ (942D, 942F). Así pues, podemos decir que, por este medio, Plutarco hace una distinción entre sus personajes principales (Lamprias y Lucio), cuyas opiniones comparte, y los secundarios (Aristóteles, Fárnaces, Apolónides, Menelao y Teón). Pero veamos ya los gestos y actitudes que generan las palabras de estos interlocutores.

En este ambiente intelectual, mezcla de rivalidad y politesse, no faltan algunos gestos que denuncian la posición enfrentada entre los personajes del diálogo, los estados de ánimo de aquéllos en el transcurso de la conversación y las actitudes por las que intervienen, se dirigen a determinados compañeros de diálogo o dejan la argumentación en manos de otros. Normalmente estos matices se señalan con participios, que nos dan el tono bajo el cual debemos interpretar los contenidos expresados por las palabras.

Entre estos participios tenemos los que indican la mayor o menor brusquedad, impaciencia, con que el personaje entra en el diálogo: $\dot{\pi} \pi \mathrm{\alpha} \alpha \beta \omega \dot{\nu}(920 \mathrm{~F}$ y 935C, Apolónides; 929E y 940F, Sila) y el participio absoluto ÉTl ( $\delta \epsilon ́) \mu O v \lambda \hat{\epsilon}-$ yovтos (922E, Fárnaces y 940F, Sila); los que expresan la búsqueda de un destinatario concreto entre los interlocuto-

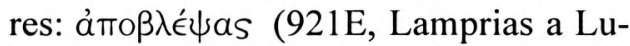

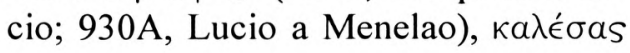

(923F, Lamprias a Lucio), los que deno-

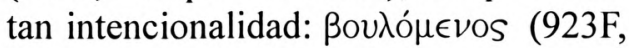
Lamprias); o estados de ánimo (complacencia, ironía, burla): jं $\sigma \theta \in$ is (921B, Apolónides) $\gamma \in \lambda a ́ \sigma a s ~(922 F$, Lucio) y $\mu \in ı \delta$ iáoas (928D, Aristóteles; 935E,

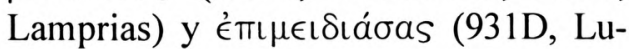
cio); hay finalmente expresiones que indican actitudes como la cesión de la palabra a otro compañero: $\epsilon \hat{\imath} \tau \alpha$ Toṽ A Sov trapévTos (933F, Apolónides a Fárnaces) o la manifestación de respeto o reconocimiento de la competencia del in-

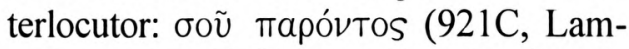
prias de Apolónides y 930A, Lucio respecto de Menelao). Estos indicadores de circunstancias van a menudo en participio, porque se ligan a la conducta o actitud de los interlocutores introducidos por el narrador con $\epsilon \hat{\imath} \pi \epsilon$, misma función desempeñan expresiones verbales con que se describe el comportamiento de los personajes antes de tomar la palabra o cuando ya están en el uso de ella. En este caso tenemos $933 \mathrm{~F}$

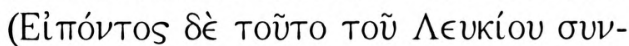

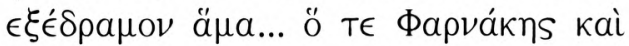

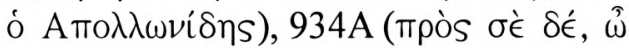

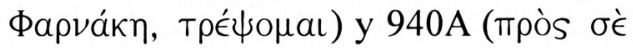

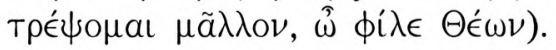

\section{4}

En definitiva, los elementos teatrales - pese a la parca escenografía del diálogono faltan en esta discusión científica que cobra vivacidad gracias a las actitudes de sus interlocutores, manifestadas en pequeños detalles, gestos tal vez y palabras. 
1) La airada indignación de Fárnaces con Lamprias y Lucio, representantes de la Academia, tiene su expresión más clara en la brusca interrupción a Lamprias y en el tono de sus palabras $(922 \mathrm{E}-$ F), en la irónica propuesta de Lamprias,

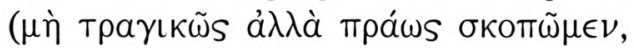
926C), en la precipitación con que, dándose por aludido, busca la palabra al mismo tiempo que (por ser tema de su competencia) Apolónides ( $\sigma v \nu \in \xi \in ́ \delta \rho a-$ $\mu o v, 933 \mathrm{~F}$ ); de hecho, no creo que sea sino el carácter vehemente del estoico, la razón que lleva a Plutarco (en palabras de Lamprias) a precisar que Apolónides le deja la preferencia en el uso de

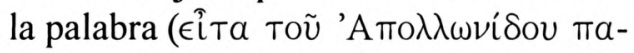

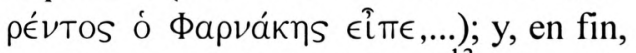
en el enrojecimiento (de ira) ${ }^{13}$ y los más que probables gestos violentos denunciados por Lamprias, cuando teme que

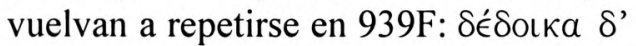

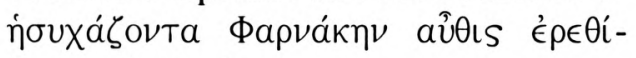

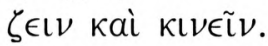

2) Más moderado el peripatético Aristóteles, no es indignación, sino enfado disimulado lo que lo lleva a irrumpir al final de la intervención de Lucio para protestar porque se haya dejado de lado en la discusión la doctrina aristotélica (928D). Su gesto, el esbozo de una sonrisa, no hace sino ocultar ese enfado.
Pero se da cuenta de este detalle Lucio y trata de justificar la omisión, atribuyéndola a las dificultades para aplicar la doctrina a la astronomía lunar, y de suavizar la actitud de Aristóteles con apelativos cariñosos (más que irónicos en este

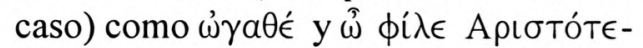
$\lambda \in S$; en cierto modo, las palabras finales de Lucio, excusando la doctrina de Anaxágoras sobre que el sol ilumina la lu-

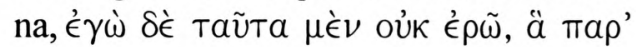

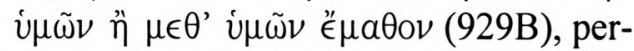
siguen el mismo efecto mediante el reconocimiento de la competencia física de los peripatéticos o la creación de una cierta camaradería de escuela.

3) La actitud de los académicos (Lucio, aunque pitagórico, se comporta como tal en todo el diálogo) hacia los estoicos es más refinada, pero no menos hiriente, que la rudeza de Fárnaces. Casi todas las referencias y los gestos de Lamprias y de Lucio hacia ellos están cargados de ironía, burla, sarcasmo o menosprecio. En primer lugar, la decisión de Lamprias de pasar directamente, tras la teoría de Clearco, a exponer la

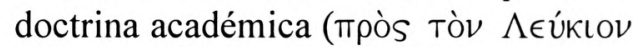

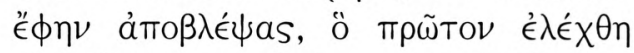
$\tau \tilde{\omega} \nu$ i $\mu \in T \epsilon ́ \rho \omega \nu$ imó $\mu \nu \eta \sigma o v, 921 \mathrm{E})$, demuestra un desprecio total por la posición de los estoicos. De hecho, la iróni-

Es éste un tema literario muy del gusto de los admiradores de Platón como Plutarco. El modelo se encuentra en los enfrentamientos de Sócrates con algunos sofistas. Por ejemplo, el enrojecimiento de ira es un síntoma que manifiesta varias veces el Trasímaco del libro I de la República, personaje especialmente bronco y agresivo en la caracterización platónica (debo la observación a Raúl Caballero). 
ca cortesía de Lucio como única motivación para contar lo que se dijo sobre esta teoría, ratifica que lo que menos interesa es su contenido dogmático. Está claro que tanto la intervención de Lamprias, como la justificación de Lucio, son un mero pretexto para introducir en sus justos términos (burla e ironía) la actitud de los académicos hacia sus contrincantes; pues, en realidad, casi toda la argumentación expuesta por ambos amigos es un debate contra Fárnaces y sus correligionarios. Actitudes, gestos y palabras, en este debate, convergen para alimentar la tensión entre Lamprias/Lucio y Fárnaces. Los gestos son la risa con que Lucio responde a la airada intervención de Fárnaces, risa seguida de provocación (el juicio a Aristarco, 922F) y las miradas o señalamientos que posiblemente encierran los oîtol con que Lamprias se refiere a los estoicos a menudo en su exposición. En cuanto a las palabras, son ataques directos, ironías, chistes, interrogaciones y exclamaciones con que se carga la tensión y se pretende ridiculizar los principios del estoicismo. Y las actitudes encuentran la horma de su zapato en esos adjetivos 'cariñosos', aparentemente 'afectuosos' con que se acompañan las interpelaciones a Fárnaces y que pierden su valor real por la carga negativa del contexto en que se emplean.

4) Pero también puede encerrar la sonrisa cierta ironía. Así ocurre con la que sirve de respuesta a Lamprias frente a las palabras de autoestima, rayanas en el engreimiento, con que Apolónides luce su competencia matemática frente a los aca-

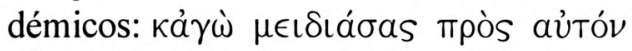

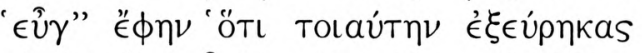

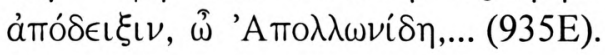

5) Es complicidad y autocomplacencia, en cambio, lo que lleva a Lucio a esbozar otra sonrisa por las palabras elogiosas de su amigo Lamprias (931D); y seguramente un gesto denuncia el placer que siente Apolónides ante la explica-

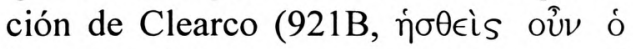
'A $\pi 0 \lambda \lambda \omega \nu i \delta \eta s)$. La aprobación, o el agradecimiento por una palabra de ayuda, se traduce igualmente en gestos, como el aplauso de todos a Lucio en $931 \mathrm{D}$, o en palabras, como las de Lucio a Lamprias en 932D (ỏ $\beta \tilde{\omega} s$ imé $\mu \nu \eta$ -

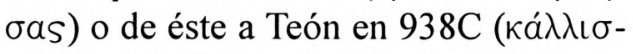

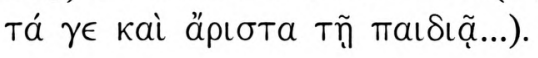

6) También está el respeto y el reconocimiento de la competencia a especialistas en campos concretos, como Apolónides y Menelao en la matemática y Teón en la filología. A Apolónides le reconoce esta competencia Lamprias en tres ocasiones: Cuando le dice que a él es a quien más corresponde conocer la doctrina del reflejo de Clearco: mavtì $\mu \tilde{a} \lambda \lambda \circ \nu^{\prime}$ '

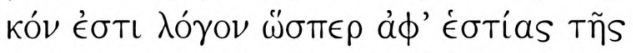
$\gamma \in \omega \mu \epsilon \tau$ pías ó $\rho \mu \omega \mu \in v o \nu, 920 \mathrm{E}$; la segunda, cuando manifiesta su reparo a dar delante de él explicaciones ópticas

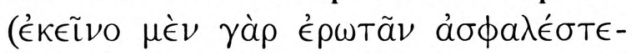

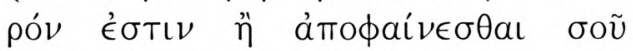
тарóvтos, 921C); y la tercera, cuando le reconoce su competencia a propósito de la excentricidad de la Luna: Toutì $\mu e ̀ v$ 


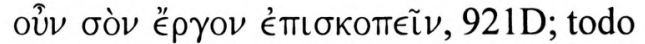
esto, sin contar las frecuentes apostillas ( que introduce Lamprias cuando utiliza conceptos o principios de las matemáticas. A Menelao le reconoce también su competencia matemática Lucio en $930 \mathrm{~A}$, cuando aquél dirige su mirada hacia éste y expresa humildemente su sonrojo al tratar cuestiones ópticas delante del científico:

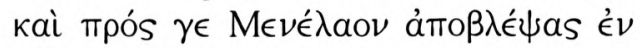

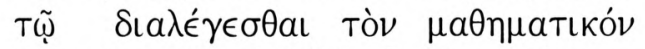

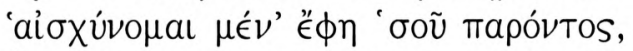

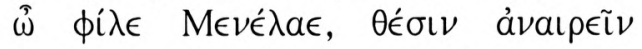

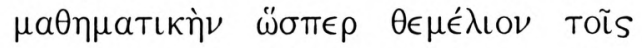

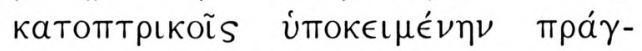

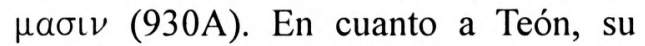
especialización filológica determina el recurso a él en varias ocasiones por parte de Lamprias y Lucio. Lamprias le pide el nombre del autor de un verso citado por él $(923 \mathrm{~F})$ o renueva su invocación en un diálogo a dos con él, para subrayar su

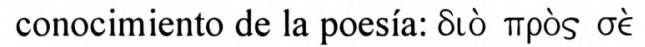

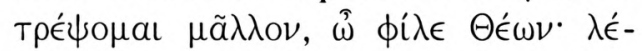

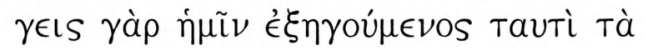
'A $\wedge \mu \alpha$ á 0 , (940A); en la misma línea, Lucio señala a Teón (como experto) a la hora de citar en su apoyo a Mimnermo, Cidias, Arquíloco, Estesícoro, Píndaro y

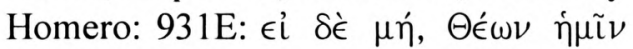

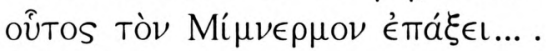

\section{5}

Terminamos ya: La riqueza de elementos dramáticos que nos hacen revivir la primera y segunda parte de este diálogo lo que hace es plasmar en el nivel de la lengua, de las estructuras sintácticas y del estilo las actitudes y posiciones filosóficas o científicas adoptadas por los personajes que intervienen en él. Y con esos elementos Plutarco marca sobre todo los motivos que determinan las intervenciones, en un par de ocasiones interrumpiendo a su interlocutor, de estos personajes. La curiosidad de Apolónides en 920F y su complacencia en 921B, la indignación de Fárnaces en 922 E-F, la reacción burlesca de Lucio en el mismo pasaje, la salida de Lamprias en ayuda de Lucio, para darle tiempo a pensar sus argumentos, en 923F, el enfado contenido de Aristóteles en 928D, la insatisfacción y curiosidad de Sila en 929E, el aplauso de Lamprias a Lucio en 931D, la curiosidad de Teón en 932 $\mathrm{D}$, la réplica profesional de Apolónides y crítica de Fárnaces en 933F, el sentido de superioridad de Apolónides en 935D y la irónica intervención de Sila en 940F, temiendo que Lamprias usurpe su puesto en la reunión, todo ello mantiene viva nuestra lectura de este diálogo. Y todo ello sirve de soporte, en la discusión filosófica, a un rico mosaico de posiciones, debates $\mathrm{e}$ ideas que afloran al texto reforzadas con gestos, palabras y actitudes que crean una tensión más o menos contenida.

Todos son conscientes en definitiva, y especialmente Lamprias, de esa tensión, que hace difícil atender tranquilamente a la sección religiosa; por ello tal vez propone a los compañeros que se tome asiento y agradece expresamente a Teón que, con la gracia de sus referencias literarias, esté contribuyendo a relajar el ceño de 
los presentes: TaũTa toũ $\Theta \epsilon ́ \omega \nu 0 S$ ei-

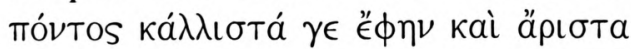

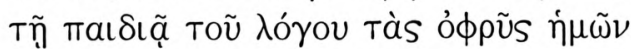

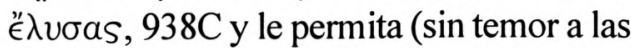
reacciones airadas de Fárnaces, a los celos de Aristóteles o al engreimiento de Apolónides), divagar por los terrenos de la hipótesis, previa a la metafísica del mito.

\section{Bibliografía CitAda}

BALDASSARI, M.,

- "Condizioni e limiti della scienza fisica nel De facie plutarcheo", en I. Gallo (ed.), Plutarco e le scienze. Atti del IV Convegno plutarcheo, Genova, 1922, pp. 267-268.

BEARDSLeE, W. A.,

- "De facie quae in orbe lunae apparet (Moralia 920A-945D)", en H. D. Betz (ed.), Plutarch's Theological Writings and Early Christian Literatur, Leiden, 1975, pp. 286-300.

Cherniss, H.,

- "Notes on Plutarch's De facie in orbe lunae", ClPh., 46 (1951) 137-158.

- "Concerning the Face which appears in the Orb of the Moon", en Plutarch's Moralia, XII, Cambridge, Loeb, 1957 (repr. 1968), pp. 1-223.

Del Corno, D.,

- "Introduzione" a Plutarco. Il volto della luna, Milano, 1991, pp. 9-41.

DoninI, P.-L.,

- "Platonism, Aristotelianism, and Stoicism in Plutarch's On the Face in the Moon", en J. Dillon \& A.A. Long (eds.), The Question of 'Eclecticism'. Studies in Later Greek Philosophy, Berkeley, Los Angeles, London, 1988, pp. 126-144.

GÖRGEMANNS, H.,

- Untersuchungen zu Plutarchs Dialog De facie in orbe lunae, Heidelberg, 1970.
KAHLE, C.,

- De Plutarchi ratione dialogorum componendorum, Diss. Gotinga, 1912.

LEHNUS, L.,

- Plutarco. Il volto della luna, Milano, 1991.

MARTIN, H. JR.,

- "Plutarch's De facie. The Recapitulations and the Lost Beginning", GRBSt, 15 (1974) 73-88.

PÉREZ JiMÉNEZ, A.,

- "Plutarco y el paisaje lunar", en J. García López \& E. Calderón Dorda (eds.), Estudios sobre Plutarco: paisaje y naturale$z a$, Madrid, 1991, pp. 307-317.

- "Ciencia, Religión y Literatura en el Mito de Sila de Plutarco", en M. Brioso \& Fco. J. González Ponce, Actitudes Literarias en la Grecia Romana, Sevilla, 1998, pp. 283-294.

Ramón Palerm, V.,

- "Sobre la cara visible de la Luna", en Plutarco. Obras morales y de costumbres (Moralia), $L X$ (Intr., trad. y notas por V. Ramón Palerm \& J. Bergua Cavero), Madrid, 2002, pp. 118-198.

TORRACA, L.,

- "L'astronomia lunare in Plutarco", en I. Gallo (ed.), Plutarco e le scienze. Atti del IV Convegno plutarcheo, Genova, 1922, pp. 231-261.

UREÑA BRACERo, J.

- "Algunos recursos de técnica dramática en los diálogos de Plutarco", en J. Fernández Delgado \& Fca. Pordomingo Pardo, Estudios sobre Plutarco. Aspectos Formales, Madrid-Salamanca, 1996, pp. 31-38.

Van Meirvenne, B.,

- “"Earth and Ambrosia' (De Facie §§ 2425): Plutarch on the Habitability of the Moon", en A. Pérez Jiménez \& F. Casadesús Bordoy, Estudios sobre Plutarco: Misticismo y religiones mistéricas en la obra de Plutarco, Madrid-Málaga, 2001, pp. 283-296. 